\title{
Fermion Localization and Resonances on A de Sitter Thick Brane
}

\author{
Yu-Xiao Liu*, Jie Yang ${ }^{\dagger}$, Zhen-Hua Zhao ${ }^{\ddagger}$, Chun-E Fu ${ }^{\S}$, Yi-Shi Duan $₫$ \\ Institute of Theoretical Physics, Lanzhou University, \\ Lanzhou 730000, People's Republic of China
}

\begin{abstract}
In arXiv:0901.3543, the simplest Yukawa coupling $\eta \bar{\Psi} \phi \chi \Psi$ was considered for a two-scalargenerated Bloch brane model. Fermionic resonances for both chiralities were obtained, and their appearance is related to branes with internal structure. Inspired on this result, we investigate the localization and resonance spectrum of fermions on a one-scalar-generated $d S$ thick brane with a class of scalar-fermion couplings $\eta \bar{\Psi} \phi^{k} \Psi$ with positive odd integer $k$. A set of massive fermionic resonances for both chiralities are obtained when provided large couple constant $\eta$. We find that the masses and life-times of left and right chiral resonances are almost the same, which demonstrates that it is possible to compose massive Dirac fermions from the left and right chiral resonances. The resonance with lower mass has longer life-time. For a same set of parameters, the number of resonances increases with $k$ and the life-time of the lower level resonance for larger $k$ is much longer than the one for smaller $k$.

PACS numbers: 11.10.Kk., 04.50.+h.
\end{abstract}

\footnotetext{
*E-mail: iuyx@lzu.edu.cn

$\dagger$ Corresponding author. E-mail: yangjiev@lzu.edu.cn

$\ddagger$ E-mail: zhaozhh02@gmail.com

$\S$ E-mail: fuche08@lzu.cn

I E-mail: ysduan@lzu.edu.cn
} 


\section{INTRODUCTION}

During the last two decades, the idea of embedding our universe in a higher dimensional space has received much attention. The suggestion that extra dimensions may not be compact [1, 2, 3, 4, 5, 6] or large [7, 8] can provide new insights for solving several puzzling phenomena such as the gauge hierarchy [8], i.e., the large difference in magnitude between the Planck and electroweak scales, the dark matter origin and the long-standing cosmological constant problem [2, 4, 9, 10, 11]. According to the brane scenarios, gravity is free to propagate in all dimensions, while all the matter fields (electromagnetic, Yang-Mills etc.) are confined to a 3-brane in a larger dimensional space. In Randall-Sundrum (RS) brane model [5], an alternative scenario of the compactification had been proposed. In this scenario, the internal manifold does not need to be compactified to the Planck scale any more, it can be large, or even infinite non-compact, which is one of the reasons why this new compactification scenario has attracted so much attention.

Recently, an increasing interest has been focused on the study of thick brane scenario in higher dimensional space-time [12, 13, 14, 15, 16, 17, 18, 19], since in more realistic models the thickness of the brane should be taken into account. In this scenario the scalar field provides a thick brane realization of the brane world as a domain wall in the bulk. For a comprehensive review on the thick brane solutions and related topics please see Ref. [20]. However, there are not so many analytic solutions of a dynamic thick domain wall. The de Sitter $(d S)$ branes have been studied in five and higher dimensional spacetimes, for examples in [16, 21, 22, 23]. The localization problem of spin half fermions on thick branes is interesting and important. The problem of fermion localization and the generation puzzle in 6D models were studied in Ref. [24]. Usually, fermions do not have normalizable zero modes in five dimensions without the scalar-fermion coupling [25, 26, 27, 28, 29, 30, 31, 32, 33, 34]. In five dimensions, with the scalar-fermion coupling, there may exist a single bound state and a continuous gapless spectrum of massive fermion Kaluza-Klein (KK) states [26, 35], while for some other brane models, there exist finite discrete KK states (mass gap) and a continuous gapless spectrum starting at a positive $m^{2}$ [36, 37]. In Ref. [32], the authors obtained trapped discrete massive fermion states on the brane, which are in fact quasi-bound and have a finite probability of escaping into the bulk. In fact, fermions can escape into the bulk by tunnelling, and the rate depends on the parameters of the scalar potential [33]. In [38], the simplest Yukawa coupling between two scalars and a spinor field was considered for a two-scalar-generated Bloch brane model [39]. Fermionic resonances for both chiralities were obtained, and their appearance is related to branes with internal structure.

In Ref. [40], we addressed the localization and mass spectra of various bulk matter fields on the $d S$ thick branes. It was shown that, all bulk matters (scalars, vectors and fermions) can be localized on these branes and the corresponding mass spectra have a mass gap. However, for spin half fermions the scalar-fermion coupling should not be the usual Yukawa coupling $\eta \bar{\Psi} \phi \Psi$ in order to trap the zero modes. In this paper, inspired on the 
results obtained in Refs. [32, 33, 38], we reinvestigate the localization problem of fermions on a one-scalar-generated $d S$ thick brane [16, 40, 41, 42, 43] with a class of scalar-fermion couplings $\eta \bar{\Psi} \phi^{k} \Psi$ with positive odd integer $k$. We shall show that, a set of massive fermionic resonances for both chiralities could be obtained when provided large couple constant $\eta$, and it is possible to compose massive Dirac fermions from the left and right chiral resonances.

The organization of the paper is as follows: In section [II, we first review the one-scalargenerated $d S$ thick brane in a 5-dimensional space-time. Then, in section III, we study localization and mass spectra of fermions on the thick brane by presenting the shapes of the mass-independent potentials of the corresponding Schrödinger problem of fermionic KK modes. We consider two different types of scalar-fermion interactions. Finally, the discussion and conclusion are given in section IV.

\section{ONE-SCALAR-GENERATED DE SITTER THICK BRANE}

Let us consider the de Sitter thick brane arising from a real scalar field $\phi$ with a scalar potential $V(\phi)$. Our system is described by the action

$$
S=\int d^{5} x \sqrt{-g}\left[\frac{1}{2 \kappa_{5}^{2}} R-\frac{1}{2} g^{M N} \partial_{M} \phi \partial_{N} \phi-V(\phi)\right],
$$

where $\kappa_{5}^{2}=8 \pi G_{5}$ with $G_{5}$ the 5 -dimensional Newton constant. Here we set $\kappa_{5}=1$. The line-element for a 5 -dimensional spacetime with planar-paralell symmetry is assumed as

$$
\begin{aligned}
d s^{2} & =\mathrm{e}^{2 A}\left(\hat{g}_{\mu \nu} d x^{\mu} d x^{\nu}+d z^{2}\right) \\
& =\mathrm{e}^{2 A}\left(-d t^{2}+e^{2 \beta t} d x^{i} d x^{i}+d z^{2}\right)
\end{aligned}
$$

where $z$ stands for the extra coordinate. The scalar field $\phi$ and the warp factor $\mathrm{e}^{2 A}$ are considered to be functions of $z$ only, i.e., $\phi=\phi(z)$ and $A=A(z)$. In the model, the potential could provide a thick brane realization. The field equations generated from the action (11) with the ansatz (2) reduce to the following coupled nonlinear differential equations

$$
\begin{aligned}
\phi^{\prime 2} & =3\left(A^{\prime 2}-A^{\prime \prime}-\beta^{2}\right), \\
V(\phi) & =\frac{3}{2} e^{-2 A}\left(-3 A^{\prime 2}-A^{\prime \prime}+3 \beta^{2}\right), \\
\frac{d V(\phi)}{d \phi} & =e^{-2 A}\left(3 A^{\prime} \phi^{\prime}+\phi^{\prime \prime}\right),
\end{aligned}
$$

where the prime denotes derivative with respect to $z$. For positive and vanishing $\beta$ one will obtain dynamic and static solutions, respectively.

A de Sitter thick brane solution in a five-dimensional spacetime for the potential

$$
V(\phi)=\frac{1+3 \delta}{2 \delta} 3 \beta^{2}\left(\cos \frac{\phi}{\phi_{0}}\right)^{2(1-\delta)}
$$


was found in Refs. [41, 42]:

$$
\begin{aligned}
e^{2 A} & =\cosh ^{-2 \delta}\left(\frac{\beta z}{\delta}\right) \\
\phi & =\phi_{0} \arctan \left(\sinh \frac{\beta z}{\delta}\right),
\end{aligned}
$$

where $\phi_{0}=\sqrt{3 \delta(1-\delta)}, 0<\delta<1, \beta>0$. The soliton configuration of the scalar field dynamically generates the domain wall configuration with warped geometry. In this system, The scalar field takes values $\pm \phi_{0} \pi / 2$ at $z \rightarrow \pm \infty$, corresponding to two consecutive minima of the potential with cosmological constant $\Lambda=0$. The scalar configuration in fact is a kink. The thick brane has a well-defined thin wall limit when $\delta \rightarrow 0$ [43] and can localize gravity and matter fields on the wall [16, 40]. For $1 / 2<\delta<1$, the hypersurfaces $|z|=\infty$ represent non-scalar spacetime singularities [16]. The energy density $\rho$ for the $d S$ brane is calculated as follows:

$$
\begin{aligned}
\rho & =3 e^{-2 A}\left(\beta^{2}-A^{2}-A^{\prime \prime}\right) \\
& =\frac{3 \beta^{2}(1+\delta)}{\delta} \cosh ^{2(\delta-1)}\left(\frac{\beta z}{\delta}\right) .
\end{aligned}
$$

\section{FERMION LOCALIZATION AND RESONANCES ON THE DE SITTER THICK BRANE}

Fermions on branes have been studied in a number of articles such as in Refs. [44, 45, 46, 47, 48, 49, 50, 51, 52, 53, 54]. In this section let us investigate whether spin $1 / 2$ fermions can be localized on the thick brane given in previous section. The Dirac action of a massless spin $1 / 2$ fermion coupled to the background scalar $\phi(8)$ is

$$
S_{1 / 2}=\int d^{5} x \sqrt{-g}\left(\bar{\Psi} \Gamma^{M}\left(\partial_{M}+\omega_{M}\right) \Psi-\eta \bar{\Psi} F(\phi) \Psi\right) .
$$

The non-vanishing components of the spin connection $\omega_{M}$ for the background metric (2) are

$$
\omega_{\mu}=\frac{1}{2} A^{\prime} \gamma_{\mu} \gamma_{5}+\hat{\omega}_{\mu}
$$

with $\hat{\omega}_{\mu}$ the spin connection derived from the metric $\hat{g}_{\mu \nu}(x)$. Then the equation of motion is given by

$$
\left[\gamma^{\mu}\left(\partial_{\mu}+\hat{\omega}_{\mu}\right)+\gamma^{5}\left(\partial_{z}+2 A^{\prime}\right)-\eta \mathrm{e}^{A} F(\phi)\right] \Psi=0
$$

Note that the sign of the coupling $\eta$ of the spinor $\Psi$ to the scalar $\phi$ is arbitrary and represents a coupling either to kink or to anti-kink domain wall. For definiteness, we shall consider in what follows only the case of a kink coupling, and thus assume that $\eta>0$. 
By making the following general chiral decomposition:

$$
\Psi=\sum_{n}\left[\psi_{L, n}(x) f_{L, n}(z)+\psi_{R, n}(x) f_{R, n}(z)\right] \mathrm{e}^{-2 A}
$$

with $\psi_{L}=-\gamma^{5} \psi_{L}$ and $\psi_{R}=\gamma^{5} \psi_{R}$ the left-handed and right-handed components of a 4D Dirac field respectively, and demanding $\psi_{L, R}$ satisfy the $4 \mathrm{D}$ massive Dirac equations $\gamma^{\mu}\left(\partial_{\mu}+\hat{\omega}_{\mu}\right) \psi_{L, R}=m \psi_{R, L}$, we obtain the following coupled equations

$$
\begin{aligned}
& {\left[\partial_{z}+\eta \mathrm{e}^{A} F(\phi)\right] f_{L}(z)=m f_{R}(z)} \\
& {\left[\partial_{z}-\eta \mathrm{e}^{A} F(\phi)\right] f_{R}(z)=-m f_{L}(z)}
\end{aligned}
$$

which can be reduced to the Schrödinger-like equations for the wavefunctions of left and right chiral fermions

$$
\begin{aligned}
& \left(-\partial_{z}^{2}+V_{L}(z)\right) f_{L}=m^{2} f_{L} \\
& \left(-\partial_{z}^{2}+V_{R}(z)\right) f_{R}=m^{2} f_{R}
\end{aligned}
$$

where the effective potentials are given by

$$
\begin{aligned}
V_{L}(z) & =\left(\eta \mathrm{e}^{A} F(\phi)\right)^{2}-\partial_{z}\left(\eta \mathrm{e}^{A} F(\phi)\right), \\
V_{R}(z) & =\left.V_{L}(z)\right|_{\eta \rightarrow-\eta} .
\end{aligned}
$$

We have dropped the index $n$ for convenience.

It can be seen that, in order to localize left or right chiral fermions, there must be some kind of scalar-fermion coupling, and the effective potential $V_{L}(z)$ or $V_{R}(z)$ should have a minimum at the location of the brane. Furthermore, for the kink configuration of the scalar $\phi(z)(8), F(\phi(z))$ should be an odd function of $\phi(z)$ when one demands that $V_{L, R}(z)$ are invariant under $Z_{2}$ reflection symmetry $z \rightarrow-z$. Thus we have $F(\phi(0))=0$ and $V_{L}(0)=-V_{R}(0)=-\eta \partial_{z} F(\phi(0))$, which results in the well-known conclusion: only one of the massless left and right chiral fermions could be localized on the brane. The spectra are determined by the behavior of the potentials at infinity. For $V_{L, R} \rightarrow 0$ as $|z| \rightarrow \infty$, one of the potentials would have a volcano-like shape and there exists only a bound massless mode followed by a continuous gapless spectrum of KK states, while another could not trap any bound states and the spectrum is also continuous and gapless. For $V_{L, R} \rightarrow V_{\infty}=$ const as $|z| \rightarrow \infty$, those modes with $m_{n}^{2}<V_{\infty}$ belong to discrete spectrum and modes with $m_{n}^{2}>V_{\infty}$ contribute to continuous one. If the potentials increase as $|z| \rightarrow \infty$ the spectra are discrete.

The concrete behavior of the potentials is depended on the function $F(\phi)$. In Ref. [40], two cases $F(\phi)=\phi$ and $F(\phi)=\sin \left(\frac{\phi}{\phi_{0}}\right) \cos ^{-\delta}\left(\frac{\phi}{\phi_{0}}\right)$ were investigated in detail as examples. For the first example with usual Yukawa couple $\eta \bar{\Psi} \phi \Psi$, there exists no mass gap but a continuous gapless spectrum of KK states. For the second example with a positive coupling constant $\eta$, there exist some discrete bound KK modes and a series of continuous ones. The 
total number of bound states increases with the couple constant $\eta$. If $0<\eta<\beta / \delta$, there is only one left chiral fermion bound state which is just the left chiral fermion zero mode; if $\eta>\beta / \delta$, there are $N_{\max }+1$ left chiral fermion bound states (including zero mode and massive KK modes) and $N_{\max }$ right chiral fermion bound states (including only massive KK modes). Especially, the spectrum of left chiral fermions

$$
\begin{aligned}
& m_{L, n}^{2}=\frac{\beta}{\delta^{2}}(2 \delta \eta-n \beta) n \\
& \left(\eta>0, n=0,1,2, \ldots, N_{\max }<\frac{\delta \eta}{\beta}\right)
\end{aligned}
$$

and right one

$$
\begin{aligned}
& m_{R, n}^{2}=\frac{\beta}{\delta^{2}}[2 \delta \eta-(n+1) \beta](n+1) \\
& \left(\eta>\frac{\beta}{\delta}, n=0,1,2, \ldots, N_{\max }-1<\frac{\delta \eta}{\beta}-1\right)
\end{aligned}
$$

are the same except the massless mode, i.e., $m_{L, n+1}^{2}=m_{R, n}^{2}$. In this paper, we would like to consider the case $F(\phi)=\phi^{k}$ with $k$ a positive odd integer, for the purpose of investigating resonances of massive fermions.

\section{A. Case I: $F(\phi)=\phi$}

Firstly, we reconsider the simplest case $F(\phi)=\phi$ for the $d S$ brane world solution (6) (6) (8) The explicit forms of the potentials (16) are

$$
\begin{aligned}
V_{L}(z)= & \eta^{2} \phi_{0}^{2} \frac{\arctan ^{2} \sinh \left(\frac{\beta z}{\delta}\right)}{\cosh ^{2 \delta}\left(\frac{\beta z}{\delta}\right)}-\frac{\eta \beta \phi_{0}}{\delta \cosh ^{1+\delta}\left(\frac{\beta z}{\delta}\right)} \\
& +\eta \beta \phi_{0} \frac{\sinh \left(\frac{\beta z}{\delta}\right) \arctan \sinh \left(\frac{\beta z}{\delta}\right)}{\cosh ^{1+\delta}\left(\frac{\beta z}{\delta}\right)} \\
V_{R}(z)= & \left.V_{L}(z)\right|_{\eta \rightarrow-\eta} .
\end{aligned}
$$

The values of the potentials (19a) and (19b) at $z=0$ and $z \rightarrow \pm \infty$ are given by

$$
\begin{aligned}
& V_{L}(0)=-V_{R}(0)=-\beta \eta \sqrt{3\left(\delta^{-1}-1\right)}, \\
& V_{L, R}(z \rightarrow \pm \infty) \rightarrow 0,
\end{aligned}
$$

i.e., both potentials have same asymptotic behavior when $z \rightarrow \pm \infty$, but opposite behavior at the origin $z=0$. In this paper we take $\beta=\delta$ and $\eta>0$ for simplicity. The shapes of the potentials are shown in Figs. 1 and 2. It can be seen that, for any $0<\delta<1$ and $\eta>0$, $V_{L}(z)$ is indeed a modified volcano type potential. Hence, the potential of left chiral fermions provides no mass gap to separate the zero mode from the excited KK ones, and there exists 
a continuous gapless spectrum of the KK modes for left chiral fermions. However, the zero mode of the left chiral fermions

$$
f_{L 0}(z) \propto \exp \left(-\eta \int^{z} d \bar{z} \mathrm{e}^{A(\bar{z})} \phi(\bar{z})\right)
$$

is non-normalizable [40], which is an example that negative value of potential at the brane location does not guarantee the existence of a normalized zero mode. This is different from the situation in Refs. [32, 46], where the corresponding potential of left chiral fermions is also a volcano type one and the zero mode can be localized on the branes provided strong enough Yukawa coupling.
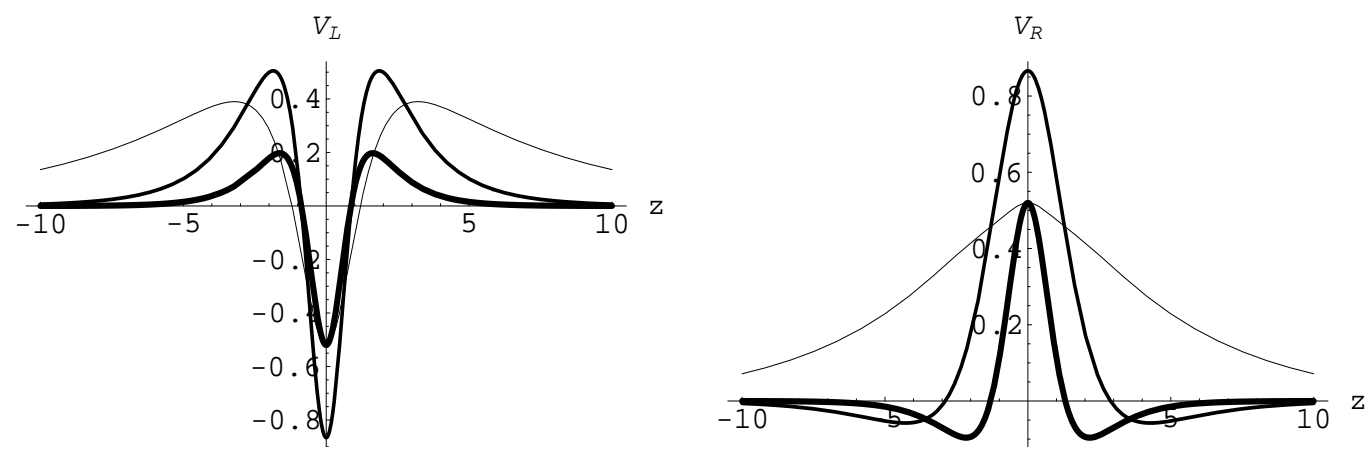

FIG. 1: The potentials $V_{L, R}$ for left and right chiral fermions with $F(\phi)=\phi$. The parameters are set to $\eta=1$ and $\delta=\beta=0.1,0.5,0.9$ for lines with thickness increases with $\delta$.

The potential $V_{R}$ is always positive at the brane location and vanishes when far away from the brane. This shows that the potential could not trap any bound fermions with right chirality and there is no zero mode of right chiral fermions. This agrees with the well-known fact that massless fermions must be single-handed in brane models [30]. However, the shape of the potential is depended on the coupling constant $\eta$. With the increase of $\eta$, a potential well around the brane location would appear and the well becomes more and more deeper. The appearance of the potential well could be related to resonances or to massive fermions with a finite life-time. In Ref. [38], a similar potential and resonances for left and right chiral fermions were found but in background of two-field thick branes with internal structure. In what follows, we follow Ref. [38] and investigate the massive modes of fermions by solving numerically Eq. (15) with potentials in (19). Differently, we present another method to calculate the probability for finding the massive modes on the brane.

In order to get the solutions of KK modes $f_{L, R}(z)$ from the second order differential equations (15), we need to impose two initial conditions. The wavefunctions of a Schrödinger equation with a finite smooth potential are continuous at any position. Furthermore, considering the even parity of the potentials, we can impose two kinds of initial conditions:

$$
f(0)=c_{0}, f^{\prime}(0)=0
$$




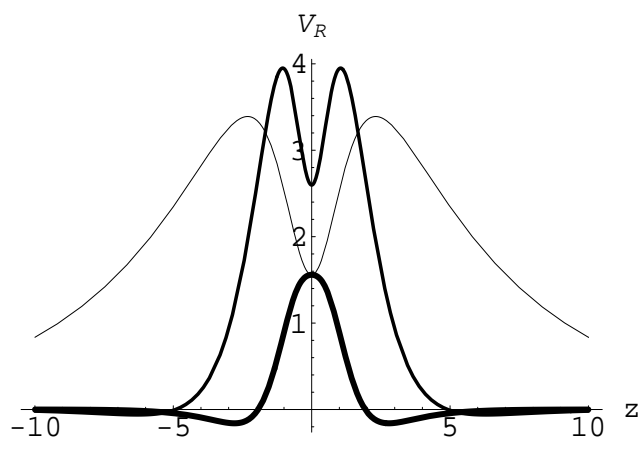

(a) $\eta=3$

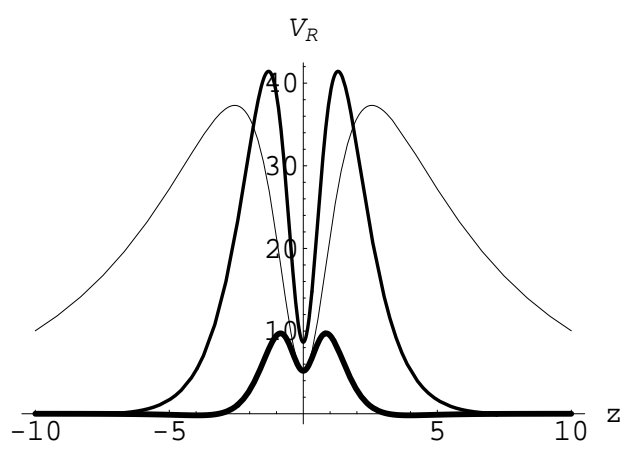

(b) $\eta=10$

FIG. 2: The potential $V_{R}$ for right chiral fermions with $F(\phi)=\phi$. The parameters are set to $\eta=3$ and $\eta=10, \delta=\beta=0.1,0.5,0.9$ for lines with thickness increases with $\delta$.

and

$$
f(0)=0, f^{\prime}(0)=c_{1}
$$

The first and second conditions would result in even and odd KK modes, respectively. The constants $c_{0}$ and $c_{1}$ for unbound massive KK modes are arbitrary but will be set to $c_{0}=1$ and $c_{1}=5$. The massive KK modes would encounter the tunneling process across the potential barriers near the brane. And the modes with different masses would have different life-times. Some massive KK modes of left chiral fermions for the case $F(\phi)=\phi$ with different $m^{2}$ are plotted in Fig. 3. These shapes show that there could exist some resonant states at some $m^{2}$. In what follows, we would like to investigate this problem.

Since Eq. (15) can be rewritten as $\mathcal{O}_{L, R}^{\dagger} \mathcal{O} f_{L, R}(z)=m^{2} f_{L, R}(z)$, one can interpret $\left|f_{L, R}(z)\right|^{2}$ as the probability for finding the massive KK modes at the position $z$ along extra dimension [38]. In Ref. [38], the authors suggested that large peaks in the distribution of $f_{L, R}(0)$ as a function of $m$ would reveal the existence of resonant states. Here we need to extend this idea for our two kinds of initial conditions (23) and (24). This is because the value of $f_{L, R}(0)$ is zero for condition (24). We proposal that large relative probabilities for finding massive KK modes within a narrow range $-z_{b}<z<z_{b}$ around the brane location, are called $P_{L, R}$, would indicate the existence of resonances. We can consider the KK modes $f_{L, R}(z)$ in a box with borders $|z|=z_{\max }$ located far from the turning point, beyond which $f_{L, R}(z)$ are turned into plane waves. The relative probabilities are defined as follows:

$$
P_{L, R}(m)=\frac{\int_{-z_{b}}^{z_{b}}\left|f_{L, R}(z)\right|^{2} d z}{\int_{-z_{\max }}^{z_{\max }}\left|f_{L, R}(z)\right|^{2} d z}
$$

where we choose $z_{b}=0.1 z_{\max }$, for which the probability for a plane wave mode with mass $m$ would be 0.1 . For these KK modes with more larger $m^{2}$ than the maximum of the corresponding potential, they would be approximatively plane waves and the probabilities 


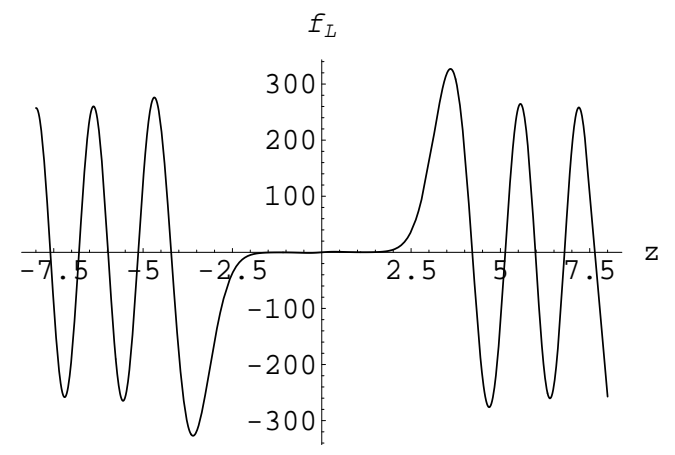

(a) $m^{2}=15.9$

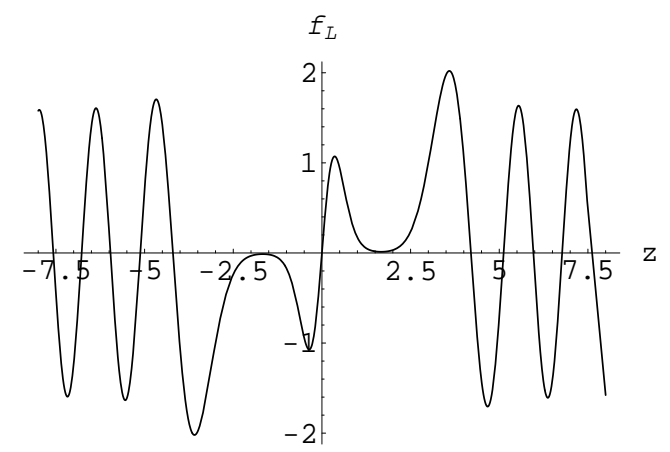

(c) $m^{2}=16.013$

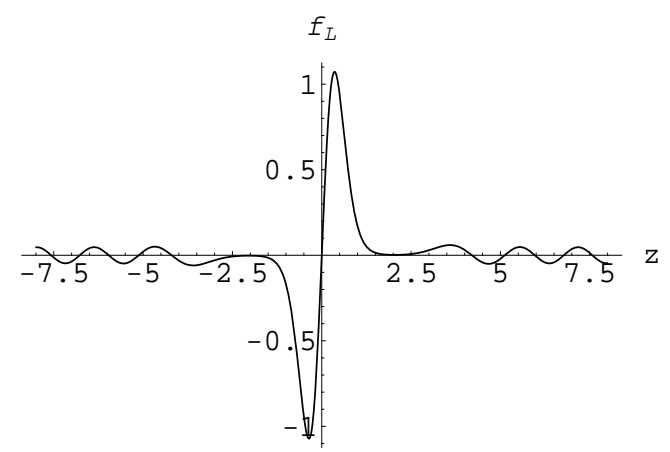

(e) $m^{2}=16.01372$

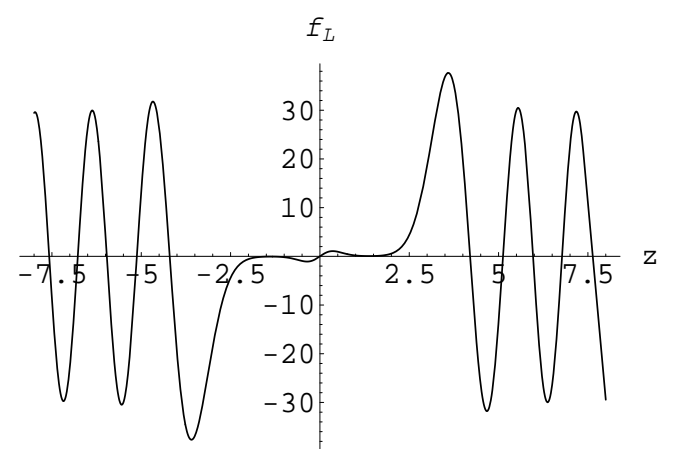

(b) $m^{2}=16$

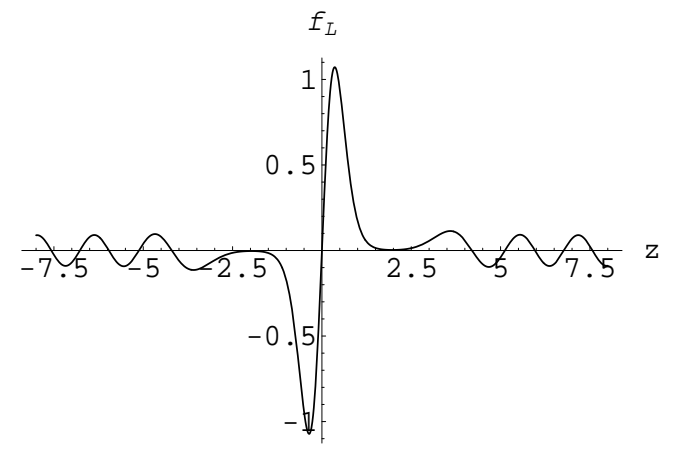

(d) $m^{2}=16.0137$

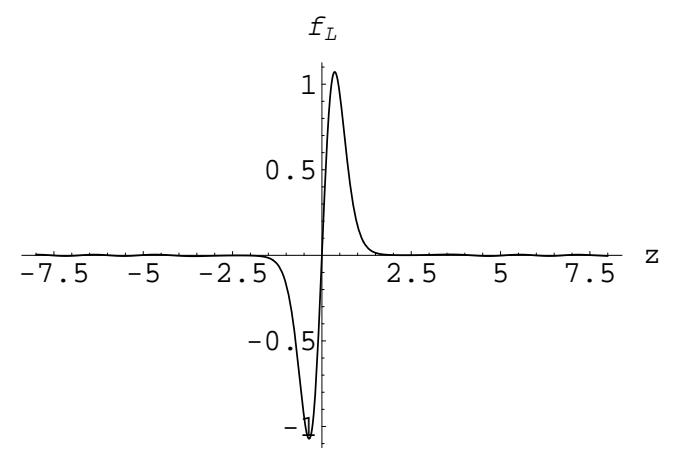

(f) $m^{2}=16.01374$

FIG. 3: Massive KK modes of left chiral fermions for the case $F(\phi)=\phi$ with different $m^{2}$. The parameters are $\delta=\beta=0.5$ and $\eta=10$.

for them would trend to 0.1 . For $\delta=\beta=0.5$ and $\eta=10$, we set $z_{\max }=30$ and the results are shown in Fig. 4. We find from the figure a series of huge peaks located at $m^{2}=16.013742,29.22241$ and 38.837314 for both left-handed and right-handed fermions. These peaks are related with resonances of fermions, which are long-lived massive fermionic 

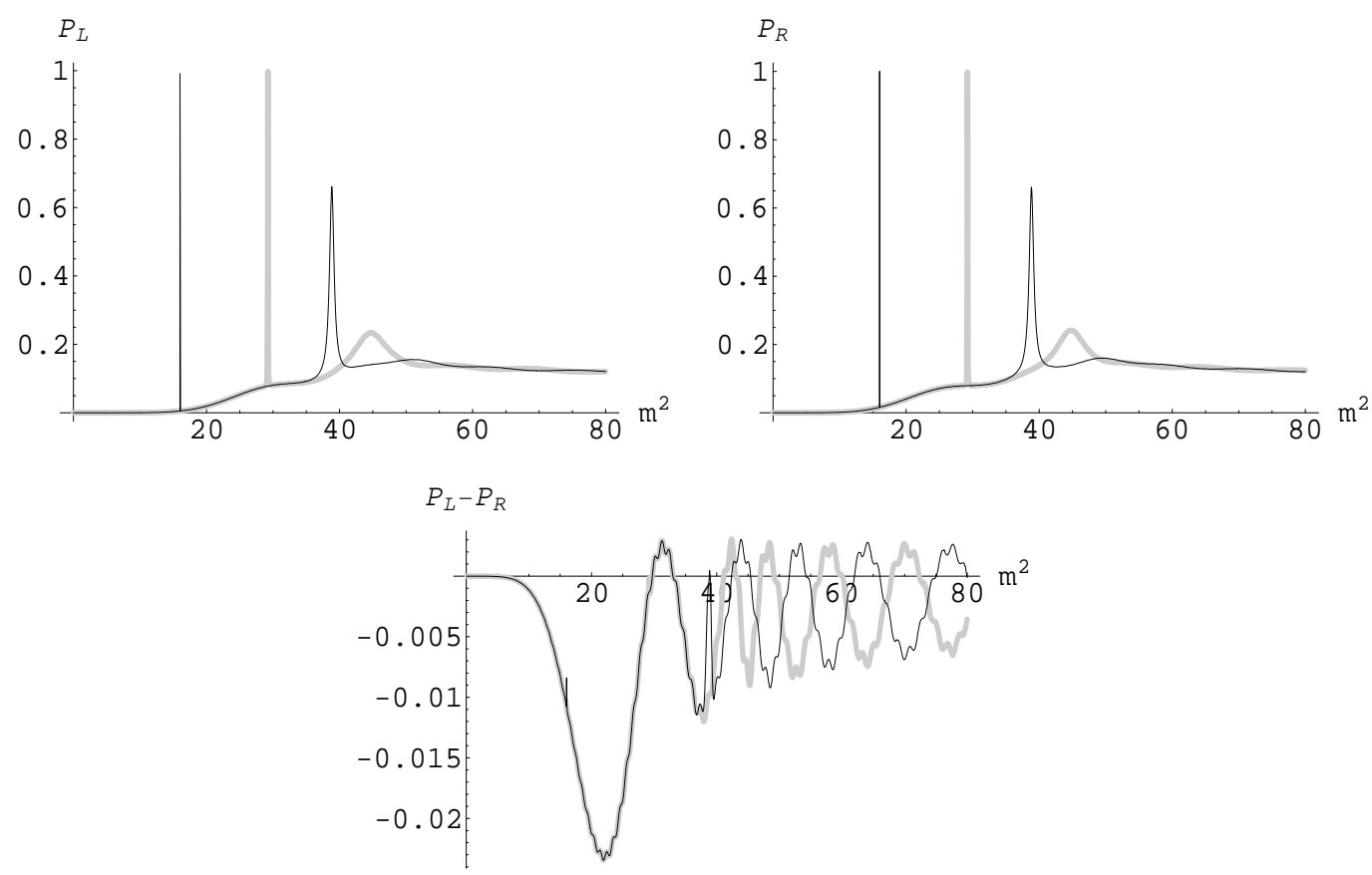

FIG. 4: The probability for finding massive KK modes of left and right chiral fermions with mass $m^{2}$ around the brane location, $P_{L, R}$, and their differences $P_{L}-P_{R}$, as a function of $m^{2}$, for the case $F(\phi)=\phi$. The parameters are $\delta=\beta=0.5$ and $\eta=10$. For $P_{L}$, gray thick lines and black thin lines are plotted for the first and second initial condition, respectively. For $P_{R}$, black thin lines and gray thick lines are plotted for the first and second initial condition, respectively.

modes on the brane. Except several peaks, the curves seemed to grow at first, and then it plateaued around $z_{b} / z_{\max }=0.1$. The reason is that, KK modes with small $m^{2}\left(\ll V_{L, R}^{\max }\right)$ will be damped near the brane and oscillate away from the brane, while those modes with large $m^{2}\left(\gg V_{L, R}^{\max }\right)$ can be approximated as plane wave modes $f_{L, R} \propto \cos m z$ or $\sin m z$.

In Figs. 5 and 6 , we plot the shapes of the resonances of left and right chiral fermions for $\delta=\beta=0.5$ and $\eta=10$. It can be seen that the configurations of Figs. $5(\mathrm{a}), 5(\mathrm{c})$ and $5(\mathrm{~d})$ could present the $n=1, n=2$ and $n=3$ level KK modes of left chiral fermions, which are in fact resonances. The $n=0$ level mode (38) with left chirality, the only one bound state, is not shown here. While the configurations of Figs. 6(a), 6(c) and 6(d) present the $n=0$, $n=1$ and $n=2$ level resonances of right chiral fermions. It is worth noting that the $n$ level massive resonance with left chirality and the $n-1$ level one with right chirality have the same mass, i.e., the spectra of massive left-handed and right-handed fermionic resonances are the same. This demonstrates that it is possible to compose a Dirac fermion from the left and right KK modes. If we only consider one of the initial conditions (23) and (24), then we could not get the resonances of left-handed and right-handed fermions with same mass at the same time. In fact, as mentioned before, we found in Refs. [37, 40] that the spectra of bound massive KK modes of left and right chiral fermions are the same, where the effective 


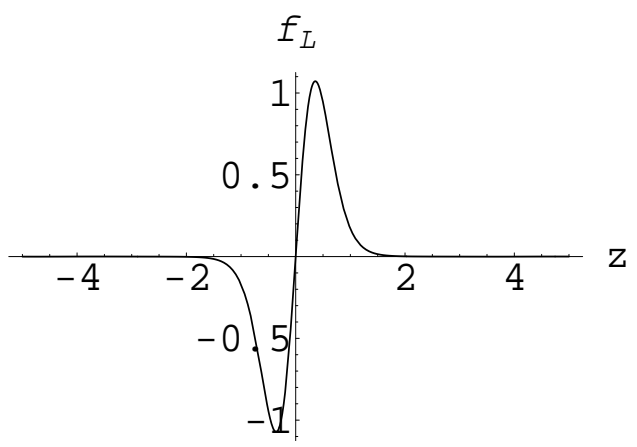

(a) $n=1, m^{2}=16.013742$

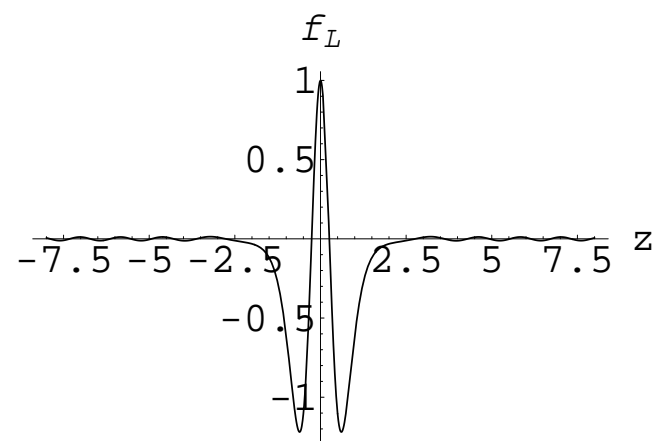

(c) $n=2, m^{2}=29.22241$

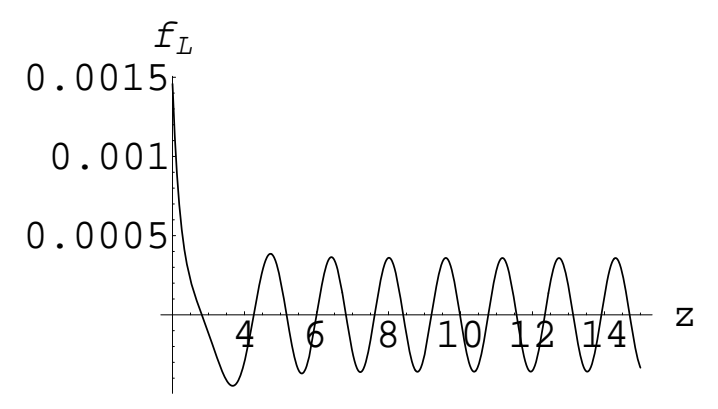

(b) Zoom in on (a)

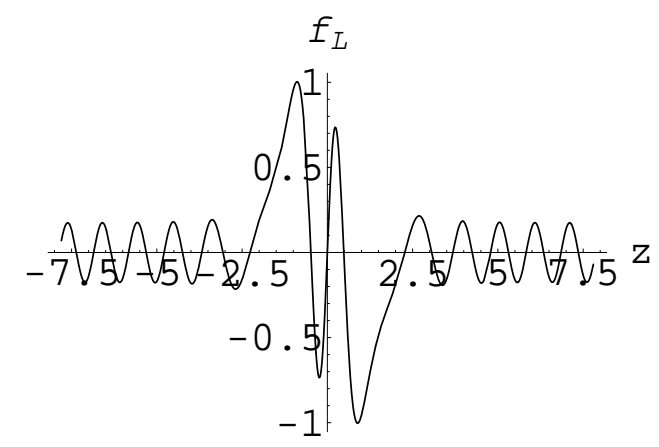

(d) $n=3, m^{2}=38.837314$

FIG. 5: Massive KK modes of left chiral fermions $f_{L}(z)$ for the case $F(\phi)=\phi$. The parameters are $\delta=\beta=0.5$ and $\eta=10$.

potentials for KK modes of fermions are modified Pöschl-Teller potentials.

We can estimate the life-time $\tau$ for a resonance from the width in mass $\Gamma=\delta m$ at half maximum of the corresponding peak in Fig. 7. This means that the fermion disappears into the extra dimension with time $\tau \sim \Gamma^{-1}$ [55]. The peaks corresponding to the resonances shown in Figs. [5 and [6 are located at $m=4.00172$, 5.40578 and 6.23196, respectively. And the width and life-time of the resonances are listed in Table I. It can be seen that the resonances with lower mass have longer life-time. And the life-times of left and right chiral resonances are almost the same, which confirms further that it is possible to compose a Dirac fermion from the left and right KK modes.

In order to get the resonances of massive KK modes for general parameters $\delta, \beta$ and $\eta$, we expand the effective potentials (19) around $z=0$ and retain terms up to order $z^{2}$. The differential equation for left chiral fermions is reduced to

$$
\left[\partial_{z}^{2}-\left(-c_{0}-m^{2}+c_{2} z^{2}\right)\right] f_{L}(z)=0
$$




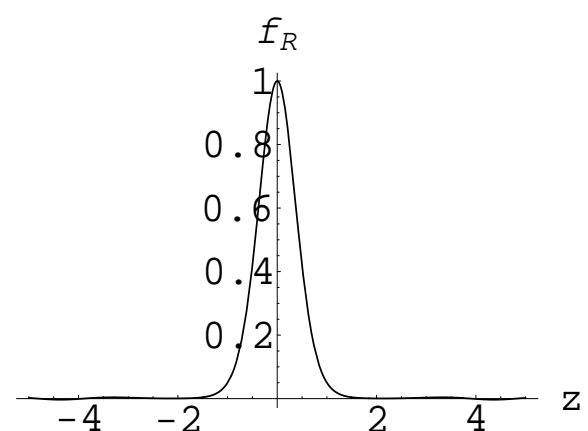

(a) $n=0, m^{2}=16.013742$

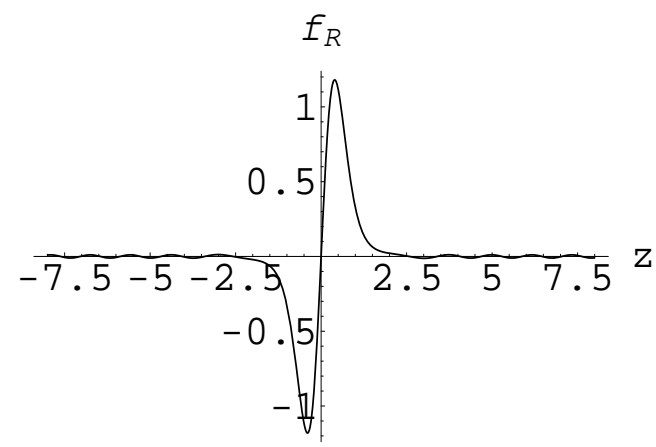

(c) $n=1, m^{2}=29.22241$

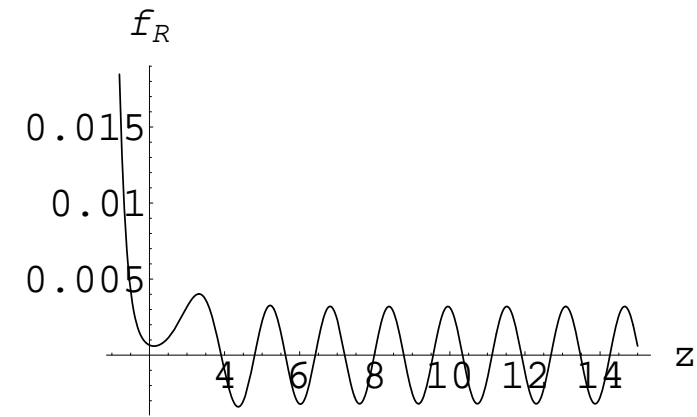

(b) Zoom in on (a)

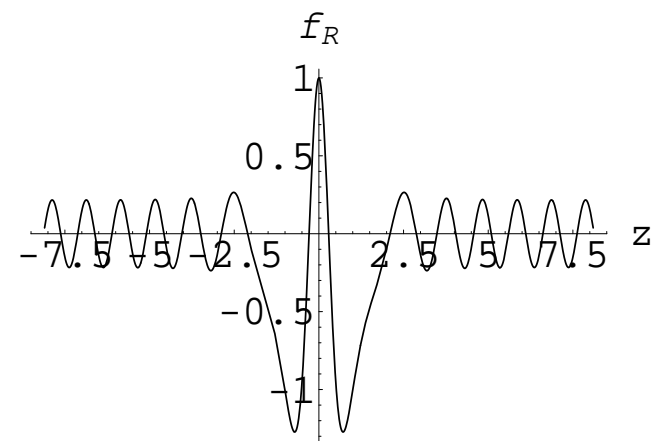

(d) $n=2, m^{2}=38.837314$

FIG. 6: Massive KK modes of right chiral fermions $f_{R}(z)$ for the case $F(\phi)=\phi$. The parameters are $\delta=\beta=0.5$ and $\eta=10$.

where

$$
\begin{aligned}
& c_{0}=\frac{\beta}{\delta} \sqrt{3(1-\delta) \delta} \eta, \\
& c_{2}=\frac{1}{2} \frac{\beta^{3}}{\delta^{3}}(1+3 \delta) \sqrt{3(1-\delta) \delta} \eta+\frac{\beta^{2}}{\delta^{2}} 3(1-\delta) \delta \eta^{2} .
\end{aligned}
$$

This is the harmonic oscillator approximation in the neighborhood of the brane and the solution is

$$
f_{L, n}(z) \propto \mathrm{e}^{-\frac{1}{2} \sqrt{c_{2}} z^{2}} H_{n}\left(c_{2}^{1 / 4} z\right)
$$

here $n=\frac{m^{2}+c_{0}}{2 \sqrt{c_{2}}}-\frac{1}{2}$ is a nonnegative integer and $H_{n}(z)$ are the Hermite polynomials. The possible values of $m^{2}$ are given by

$$
\begin{aligned}
m_{L, n}^{2}= & \frac{1+2 n}{\sqrt{2}} \sqrt{\frac{\beta^{2}}{\delta^{3}}\left(6(1-\delta) \delta^{2} \eta+\beta \sqrt{3(1-\delta) \delta}(1+3 \delta)\right) \eta} \\
& -\frac{\beta}{\delta} \sqrt{3(1-\delta) \delta} \eta \quad(n=0,1,2, \cdots) .
\end{aligned}
$$




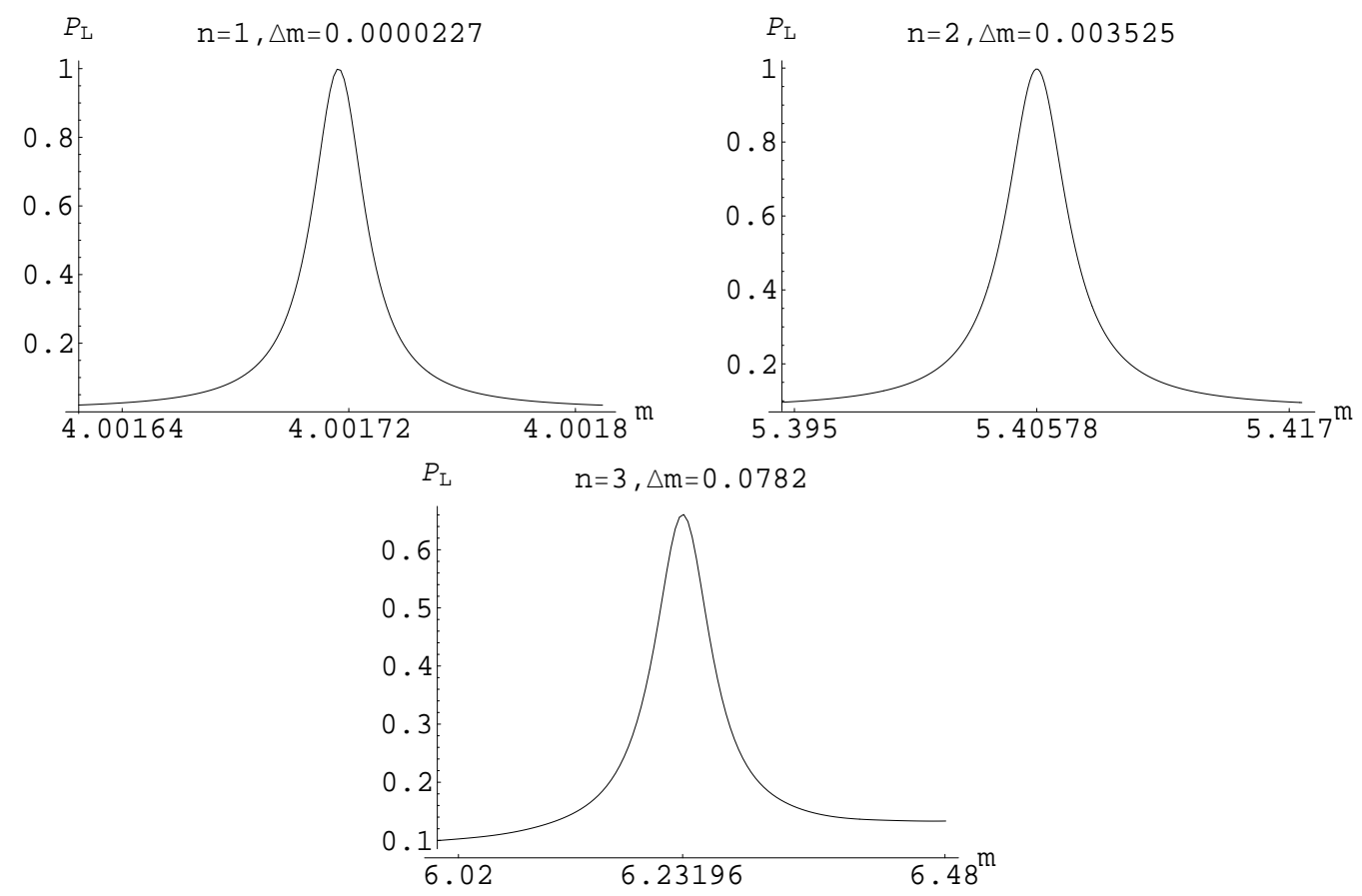

FIG. 7: The probability for finding massive KK modes of left chiral fermions around the brane location, $P_{L}$, as a function of $m$. The parameters are $\delta=\beta=0.5$ and $\eta=10$.

TABLE I: The mass, width and life-time for resonances of left and right chiral fermions. The parameters are $\delta=\beta=0.5$ and $\eta=10$.

\begin{tabular}{|l||c|c|c|c|}
\hline & $m^{2}$ & $m$ & $\Gamma$ & $\tau$ \\
\hline \hline$n=1$ (left) & 16.013742 & 4.00172 & 0.0000227 & 44052.9 \\
\hline$n=0$ (right) & 16.013742 & 4.00172 & 0.0000228 & 43859.6 \\
\hline$n=2$ (left) & 29.22241 & 5.40578 & 0.003525 & 283.688 \\
\hline$n=1$ (right) & 29.22241 & 5.40578 & 0.003534 & 282.965 \\
\hline$n=3$ (left) & 38.837314 & 6.23196 & 0.0782 & 12.7877 \\
\hline$n=2$ (right) & 38.837314 & 6.23196 & 0.0804 & 12.4378 \\
\hline
\end{tabular}

For right chiral fermions, we have

$$
\begin{aligned}
m_{R, n}^{2}= & \frac{1+2 n}{\sqrt{2}} \sqrt{\frac{\beta^{2}}{\delta^{3}}\left(6(1-\delta) \delta^{2} \eta-\beta \sqrt{3(1-\delta) \delta}(1+3 \delta)\right) \eta} \\
& +\frac{\beta}{\delta} \sqrt{3(1-\delta) \delta} \eta \quad(n=0,1,2, \cdots) .
\end{aligned}
$$

Note that, this is only an approximate solution for the spectra of left and right chiral 
fermions. For zero mode of left chiral fermions, we should have $m_{L, 0}^{2}=0$, which results in the constrained condition for the approximate solution:

$$
\eta \gg \frac{\beta \sqrt{(1-\delta) \delta}(1+3 \delta)}{2 \sqrt{3}(1-\delta) \delta^{2}} .
$$

If we take $\beta=\delta=0.5$, then we need to consider $\eta \gg 1.44$. Under the above condition, the mass spectra of fermions are reduced to the following form:

$$
\begin{aligned}
& m_{L, n}^{2}=2 \frac{\beta}{\delta} \sqrt{3(1-\delta) \delta} \eta n, \\
& m_{R, n}^{2}=2 \frac{\beta}{\delta} \sqrt{3(1-\delta) \delta} \eta(1+n) .
\end{aligned}
$$

Now, it is clear that the spectra of left and right massive fermions are the same and only lower $n$ are available. The mass of the first resonance of fermions is read as $m_{0}^{2}=2 \sqrt{3(1-\delta) \delta} \eta \beta / \delta$.

It should point out that in order to get a better approximate solution for the resonance spectra and KK modes of left and right chiral fermions, we need to solve the Schrödinger equation for the approximate potential at both large $z$ and small $z$, and match two solution to get the spectrum. In this paper, we do not discuss the question in detail.

\section{B. Case II: $F(\phi)=\phi^{k}$ with odd $k>1$}

Next we consider a simple generalization of the usual Yukawa coupling: $F(\phi)=\phi^{k}$, where $k$ is a positive odd integer. We have known in previous subsection that, for the usual Yukawa coupling $(k=1)$, the appearance of a well in the effective potential of right-handed fermions needs large couple constant $\eta$, i.e., strong kink-fermion coupling. However, the case $F(\phi)=\phi^{k}$ with $k>1$ has a very different characteristic from the $k=1$ case at the brane location. This can be seen from the derivative of $F(\phi(z))$ with respect to $z$ at $z=0$ :

$$
\partial_{z} F(\phi(0))=k \phi^{k-1}(0) \partial_{z} \phi(0) \begin{cases}\neq 0 & \text { for } k=1 \\ =0 & \text { for } k>1\end{cases}
$$

Together with $\phi(0)=\phi^{k}(0)=0$, they would result in different values of the effective potentials of fermions (16) at brane location for two kind of coupling. For the current case, the potentials (16) are now

$$
\begin{aligned}
V_{L}(z)= & \frac{\arctan ^{2 k} \sinh \left(\frac{\beta z}{\delta}\right)}{\cosh ^{2 \delta}\left(\frac{\beta z}{\delta}\right)} \phi_{0}^{2 k} \eta^{2} \\
& -\frac{\arctan ^{k-1} \sinh \left(\frac{\beta z}{\delta}\right)}{\delta \cosh ^{1+\delta}\left(\frac{\beta z}{\delta}\right)} k \phi_{0}^{k} \beta \eta \\
& +\frac{\arctan ^{k} \sinh \left(\frac{\beta z}{\delta}\right)}{\cosh ^{\delta}\left(\frac{\beta z}{\delta}\right) \operatorname{coth}\left(\frac{\beta z}{\delta}\right)} \phi_{0}^{k} \beta \eta, \\
V_{R}(z)= & \left.V_{L}(z)\right|_{\eta \rightarrow-\eta} .
\end{aligned}
$$


Both potentials have same asymptotic behavior when $z \rightarrow \pm \infty$ and $z=0$ :

$$
V_{L, R}( \pm \infty)=V_{L, R}(0)=0 .
$$

Therefore, for any positive $\eta$, there is a potential well for right-handed fermion. Especially, the potential well for left-handed fermions becomes a double-well. The shapes of the two potentials are shown in Figs. 8 and 9 for different values of $\beta, \delta, \eta$ and $k$. The depth of the potential well increases with $\beta, \eta$ and $k$, and decreases when $\delta \rightarrow 0$ or 1 . It is remarkable that, even for a very small $\eta$, there is a potential well for both left and right handed fermions. For a large $\eta$, the potential bars for both fermions are almost equal. However, for a small one, the potential bar of right-handed fermions is much higher than that of the left-handed ones.
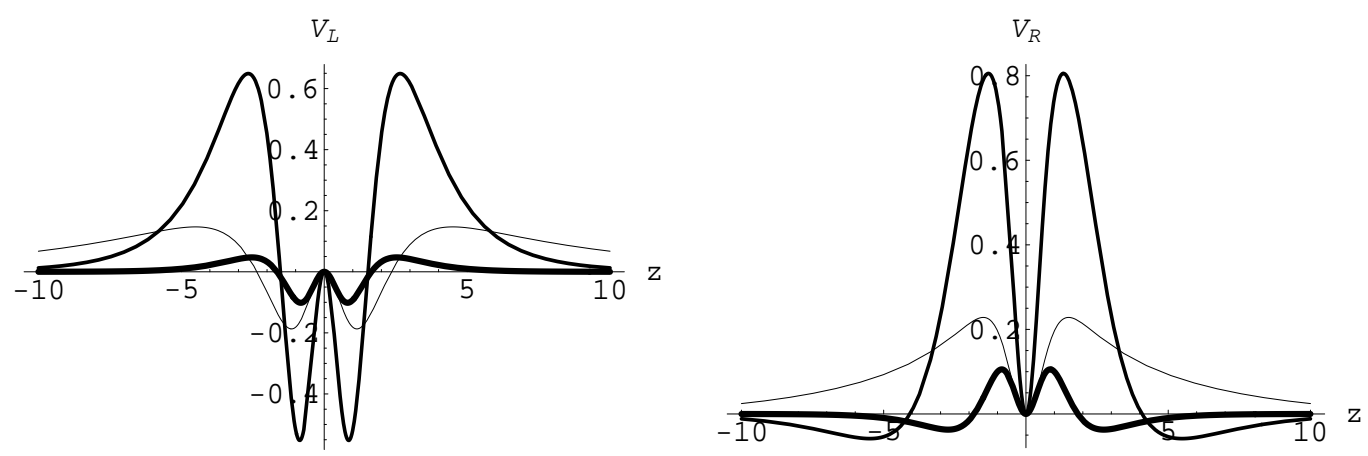

FIG. 8: The potentials $V_{L, R}$ for left and right chiral fermions with $F(\phi)=\phi^{3}$. The parameters are set to $\eta=1$ and $\delta=\beta=0.1,0.5,0.9$ for lines with thickness increases with $\delta$.
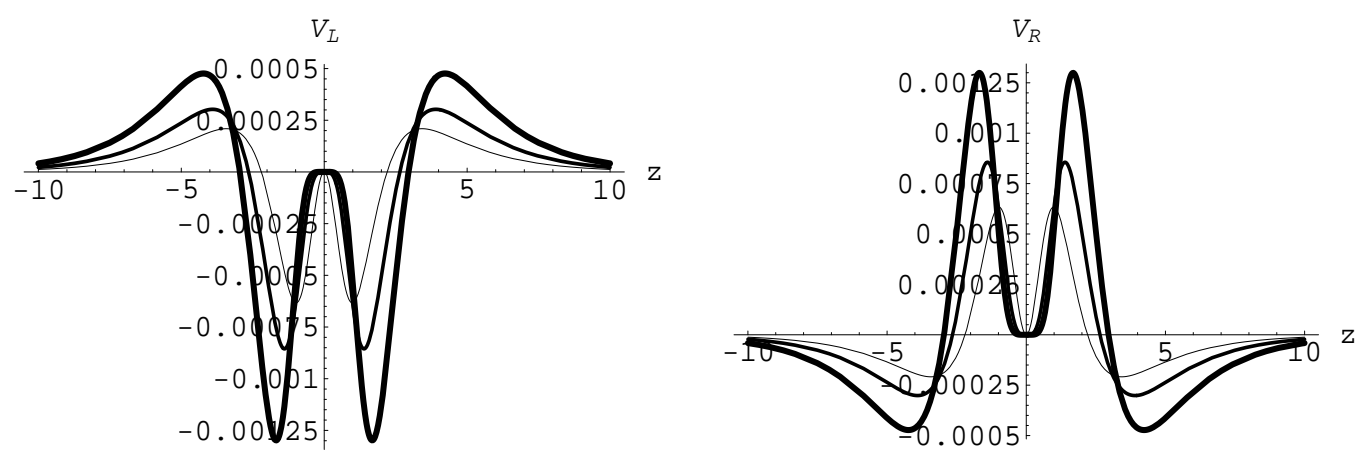

FIG. 9: The potentials $V_{L, R}$ for left and right chiral fermions with $F(\phi)=\phi^{k}$. The parameters are set to $\eta=0.001, \delta=\beta=0.5$, and $k=3,5,7$ for lines with thickness increases with $k$.

There exists a continuous gapless spectrum of the KK modes for both the left chiral and right chiral fermions. The zero mode of the left chiral fermions

$$
f_{L, 0}(z) \propto \exp \left(-\eta \int^{z} d \bar{z}^{A(\bar{z})} \phi^{k}(\bar{z})\right)
$$


is also non-normalizable. We also expand the effective potential (36b) of right chiral fermions in the neighborhood of the brane for the purpose of obtaining the resonance solutions of massive KK modes:

$$
\begin{aligned}
V_{R}= & \frac{k \beta \eta \phi_{0}^{k}}{\delta}\left(\frac{\beta z}{\delta}\right)^{k-1}-\frac{(k+2)(k+3 \delta) \beta \eta \phi_{0}^{k}}{6 \delta}\left(\frac{\beta z}{\delta}\right)^{k+1} \\
& +\mathcal{O}(z)^{k+3} . \quad(k \geq 3)
\end{aligned}
$$

Note that another term $\eta^{2} \phi_{0}^{2 k}\left(\frac{\beta z}{\delta}\right)^{2 k}$ should be included for the case $k=1$. Now we see that the case $k=3$ is special, for which the Schrödinger equation with lowest order potential can be solved analytically, and the solution is

$$
\begin{aligned}
f_{R, n} & \propto \mathrm{e}^{-\frac{1}{2} \sqrt{a} z^{2}} H_{n}\left(a^{1 / 4} z\right), \\
m_{R, n}^{2} & =(1+2 n) \sqrt{a},
\end{aligned}
$$

where $a=3 \eta(\sqrt{3(1-\delta) \delta} \beta / \delta)^{3}, n=0,1,2, \cdots$. For lowest state, we have

$$
m_{R, 0}^{2}=\sqrt{3 \eta(\sqrt{3(1-\delta) \delta} \beta / \delta)^{3}} .
$$

This is very different from the case of $k=1$, where the mass of the first resonance is decided by $m_{R 0}^{2}=2 \sqrt{3(1-\delta) \delta} \eta \beta / \delta$. Note that, we can not get the similar approximate spectrum solution for left-handed fermions for the case $k \geq 3$ because of the negative derivative of $V_{L}(z)$ at $z=0$. But we can also expect that the spectrum of lefthanded fermions is the same as that of the right-handed ones. This can be checked by numerical results (see Fig. 10). The numerical calculation shows that the spectrum (41) for large coupling $\eta$ is a good approximation. For example, for the parameters $\delta=\beta=0.5, k=3$, and $\eta=10$, the resonance spectrum calculated from the formula (41) is $m_{n}^{2}=(4.4,13.2,22.1,30.9,39.7,48.6,57.4)$, while the numerical result is $m_{n}^{2}=(4.1,12.3,20.8,29.8,38.6,46.8,53.6)$. The life-times for the $n=0,1,2,4,6$ resonances are estimated as $\tau=1.39 \times 10^{10}, 5.81 \times 10^{7}, 9.57 \times 10^{5}, 1.49 \times 10^{3}, 15.5$ respectively. It shows that the resonances with lower $n$ would have longer life-time and may be found in future high energy experiments. For small $\beta$ and $\eta$, the potential has also similar well shape, but the numerical calculation shows that there is no resonances with long life-time. This is because the depth of the well is not deep enough.

Comparing Fig. 4 with Fig. 10, we see that the number of resonances of the case $F(\phi)=\phi^{3}$ is more than that of the case $F(\phi)=\phi$ for a same set of parameters. In Table III. we list the relation of the number of the resonances $N$, the mass square $m_{N-1}^{2}$, the probability $P_{N-1}$, the width $\Delta m_{N-1}$ and the life-time $\tau_{N-1}$ of the $(N-1)$-th resonance of right-handed fermions with $k$ for the set of parameters: $\delta=\beta=0.5$ and $\eta=10$. In Fig. 11 the shapes of the $n=(N-1)$-th resonance modes of right chiral fermions for the case $F(\phi)=\phi^{k}$ are plotted. The numerical results show that the life-times of the $(N-1)$-th resonances for different $k$ are the same order. However, the life-time of the lower level (i.e., lower $n$ ) resonance for larger $k$ is much longer than the one for smaller $k$. 

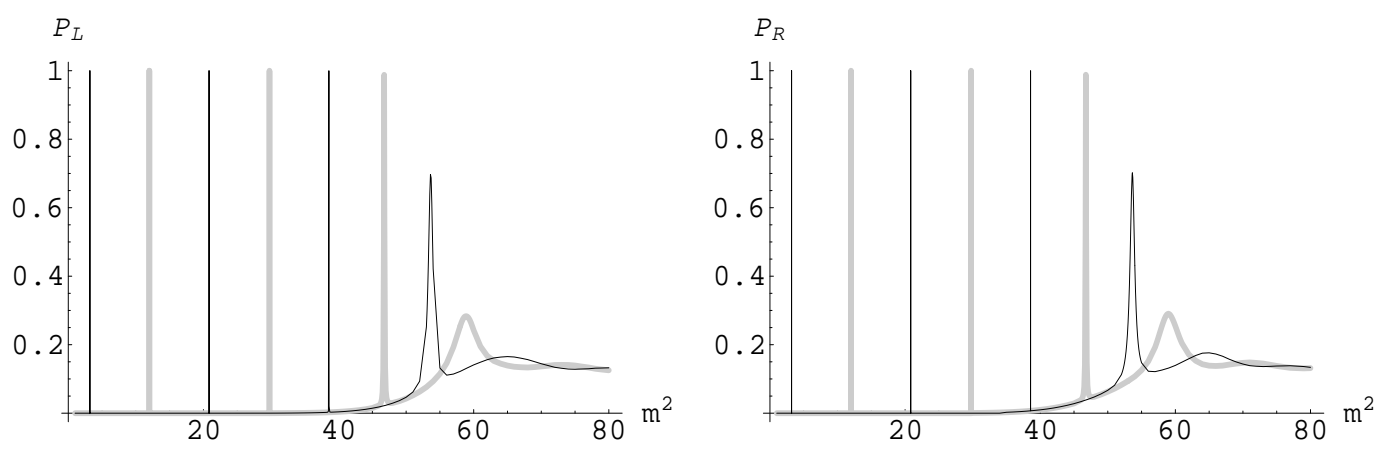

FIG. 10: The probability for finding massive KK modes of left and right chiral fermions with mass $m^{2}$ around the brane location, $P_{L, R}$, as a function of $m^{2}$, for the case $F(\phi)=\phi^{3}$. The parameters are $\delta=\beta=0.5$ and $\eta=10$. For $P_{L}$, gray thick lines and black thin lines are plotted for the first and second initial condition, respectively. For $P_{R}$, black thin lines and gray thick lines are plotted for the first and second initial condition, respectively.

TABLE II: The relation of the number of the resonances $N$, the mass square $m_{N-1}^{2}$, the probability $P_{N-1}$, the width $\Delta m_{N-1}$ and the life-time $\tau_{N-1}$ of the $(N-1)$-th resonance of right-handed fermions with $k$. The parameters are $\delta=\beta=0.5$ and $\eta=10$.

\begin{tabular}{|c||c|c|c|c|c|}
\hline$k$ & $N$ & $P_{N-1}$ & $m_{N-1}^{2}$ & $\Delta m_{N-1}$ & $\tau_{N-1}$ \\
\hline \hline 1 & 3 & 0.66 & 38.84 & 0.0782 & 12.8 \\
\hline 3 & 7 & 0.70 & 53.64 & 0.0644 & 15.5 \\
\hline 5 & 13 & 0.83 & 114.1 & 0.0361 & 27.7 \\
\hline 7 & 24 & 0.37 & 287.8 & 0.0383 & 26.1 \\
\hline 9 & 44 & 0.31 & 784.0 & 0.0443 & 22.6 \\
\hline 11 & 79 & 0.33 & 2200 & 0.0171 & 58.4 \\
\hline
\end{tabular}

\section{DISCUSSION AND CONCLUSION}

In this paper, by presenting the shapes of the mass-independent potentials of KK modes in the corresponding Schrödinger equations, we have investigated the localization and resonance spectrum of fermionic fields on a one-field generated $d S$ thick brane. It is shown that, in order to localize left or right chiral fermions on the brane, some kind of kink-fermion coupling should be introduced. A class of kink-fermion couplings $\eta \bar{\Psi} \phi^{k} \Psi$ with positive $\eta$ and positive odd integer $k$ are investigated in detail. It is worth to point out that, in this $d S$ thick brane scenario, the potential of KK modes of left chiral fermions is very different from the potentials of KK modes of the fields with spin 0, 1 and 2. For other spin fields, the potentials 


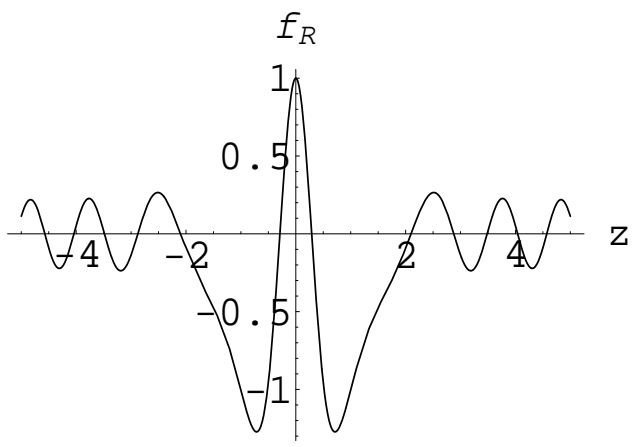

(a) $k=1, n=N-1=2$

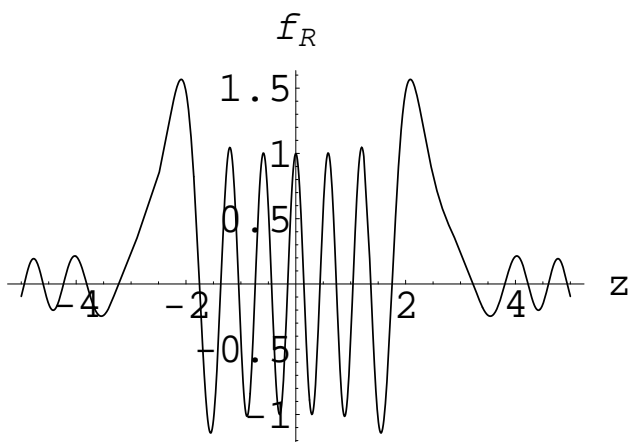

(c) $k=5, n=N-1=12$

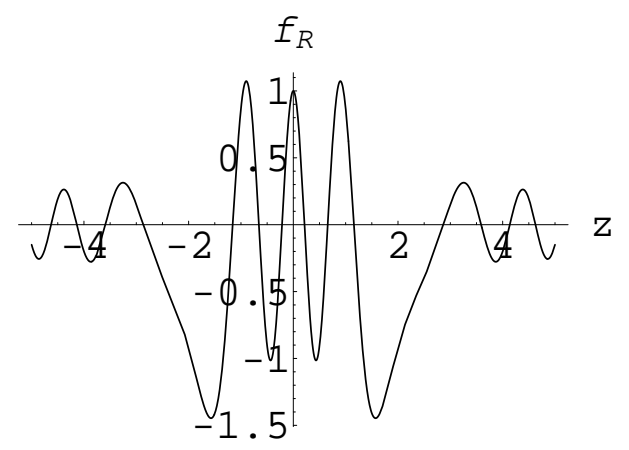

(b) $k=3, n=N-1=6$

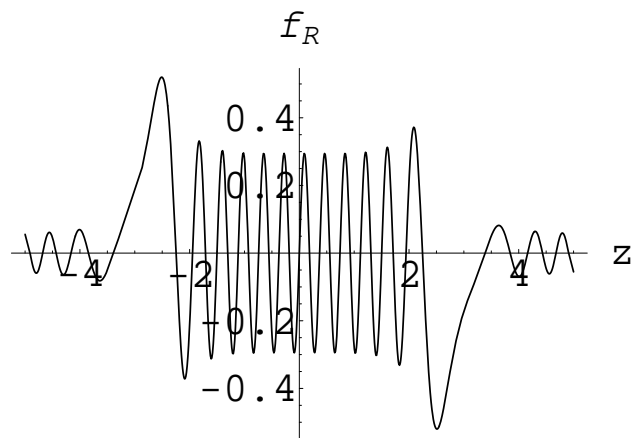

(d) $k=7, n=N-1=23$

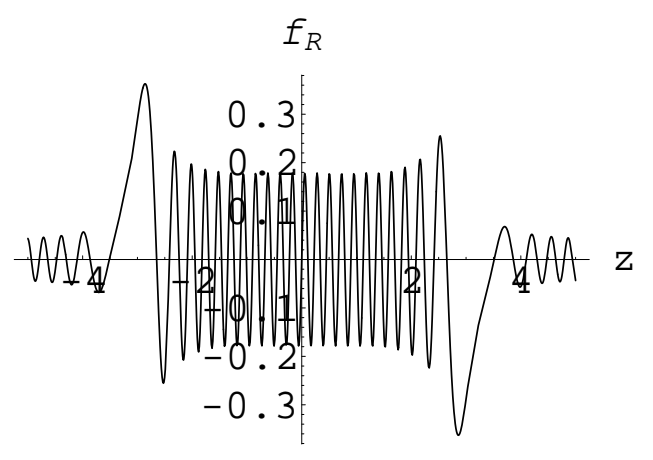

(e) $k=9, n=N-1=43$

FIG. 11: The $n=(N-1)$-th resonance modes of right chiral fermions for the case $F(\phi)=\phi^{k}$. The parameters are $\delta=\beta=0.5$, and $\eta=10$.

of KK modes are the modified Pöschl-Teller potentials, which suggest that there exist a mass gap and a series of continuous spectrum starting at positive $\mu^{2}[16,40]$. While the potential of KK modes of left chiral fermions is a modified volcano type potential. The potentials for both left and right chiral fermions vanish asymptotically when far away from the brane, 
hence all values of $\mu^{2}>0$ are allowed, and there exists no mass gap but a continuous gapless spectrum of KK states with $\mu^{2}>0$. The massive KK modes asymptotically turn into continuous plane waves when far away from the brane.

For the simplest kink-fermion coupling $\eta \bar{\Psi} \phi \Psi$, the potential of KK modes of right chiral fermions is always positive at the brane location and vanishes when far away from the brane. This shows that the potential could not trap any bound fermions with right chirality and there is no zero mode of right chiral fermions. However, with the increase of $\eta$, a potential well around the brane location appears and the well becomes more and more deeper. A set of massive fermions with a finite life-time (resonances) are obtained. We find that the masses and life-times of left and right chiral resonances are almost the same, which demonstrates that it is possible to compose a massive Dirac fermion from the left and right chiral resonances. This conclusion agrees with the case of bound massive fermionic KK modes given in Refs. [37, 40], where mass spectrums of left and right chiral massive fermions are the same.

For the case $F(\phi)=\phi^{k}$ with odd $k>1$, the appearance of a well in the effective potential of right-handed fermions does not need large couple constant $\eta$. Especially, the potential well for left-handed fermions becomes a double-well. It is also shown that the spectrum of left-handed fermionic resonances is the same as that of the right-handed ones. The resonance with lower mass has longer life-time. For a same set of parameters, the number of resonances increases with $k$ and the life-time of the lower level resonance for larger $k$ is much longer than the one for smaller $k$. For small $\beta$ and $\eta$, there is no resonances with long life-time.

At last, we give a comment on the fact that either the left or the right resonance peaks at the brane's location itself. From the coupled equations (14), it can be seen that the massive KK modes with different chirality but same mass are in fact contacted with each other. Since $e^{A}(z) F(\phi(z))$ is odd, an odd left-chiral massive KK mode will correspond to an even right one with same mass, and vice versa. This results in the fact that either the left or the right resonance peaks at the brane's location itself. So, at the brane location $z=0$, we can not get a massive resonance made of left and right chiral KK modes with same mass. This is to say, if we allow measurements only at $z=0$, the massive resonances found on the brane are all chiral, and the evidence of Dirac fermions is lost. However, in realistic thick brane models, branes are extended objects along the extra dimension. So we can interpret the probability for finding the massive modes on the brane (not necessarily at $z=0)$ as $\int_{-z_{b}}^{z_{b}} d z\left|f_{L, R}(z)\right|^{2}$ or more suitable as (25), where the parameter $z_{b}$ is chosen in order to allow the influence of the odd modes in small regions around the brane location $z=0$. According to this idea, the formation of metastable massive Dirac fermions is realized, and those fermions are quasi-localized on the brane. Similar discussions can also be found in Refs. [27, 38, 56, 57, 58] for massive fermions or gravitons. 


\section{Acknowledgement}

Y.X. Liu would like to thank Adalto R. Gomes for useful discussions, and the authors thank the referees for remarks that improved the work. This work was supported by the Program for New Century Excellent Talents in University, the National Natural Science Foundation of China(NSFC)(No. 10705013), the Doctoral Program Foundation of Institutions of Higher Education of China (No. 20070730055), the Key Project of Chinese Ministry of Education (No. 109153) and the Fundamental Research Fund for Physics and Mathematics of Lanzhou University (No. Lzu07002).

[1] K. Akama, Pregeometry, in Proceedings of the "Symposium on Gauge Theory and Gravitation", Nara Japan August 20-24 1982, K. Kikkawa, N. Nakanishi and H. Nariai eds., Springer-Verlag, Germany (1983), page 267-271 [Lect. Notes Phys. 176 (1982) 267], arXiv:hep-th/0001113.

[2] V.A. Rubakov and M.E. Shaposhnikov, Phys. Lett. B 125 (1983) 136; V.A. Rubakov and M.E. Shaposhnikov, Phys. Lett. B 125 (1983) 139; E.J. Squires, Phys. Lett. B 167 (1986) 286.

[3] M. Visser, Phys. Lett. B 159 (1985) 22.

[4] S. Randjbar-Daemi and C. Wetterich, Phys. Lett. B 166 (1986) 65.

[5] L. Randall and R. Sundrum, Phys. Rev. Lett. 83 (1999) 3370; Phys. Rev. Lett. 83 (1999) 4690.

[6] J. Lykken and L. Randall, JHEP 0006 (2000) 014.

[7] I. Antoniadis, Phys. Lett. B 246 (1990) 377.

[8] N. Arkani-Hamed, S. Dimopoulos and G. Dvali, Phys. Lett. B 429 (1998) 263; I. Antoniadis, N. Arkani-Hamed, S. Dimopoulos and G. Dvali, Phys. Lett. B 436 (1998) 257.

[9] N. Arkani-Hamed, S. Dimopoulos, N.Kaloper and R. Sundrum, Nucl. Phys. B 480 (2000) 193; S. Kachru, M. Schulz and E. Silverstein, Phys. Rev. D 62 (2000) 045021; A. Kehagias, Phys. Lett. B 600 (2004) 133.

[10] G.D. Starkman, D. Stojkovic and M. Trodden, Phys. Rev. D 63 (2001) 103511; Phys. Rev. Lett. 87 (2001) 231303.

[11] Y. Shtanov, V. Sahni, A. Shafieloo and A. Toporensky, JCAP 04 (2009) 023, arXiv:0901.3074[gr-qc].

[12] O. DeWolfe, D.Z. Freedman, S.S. Gubser and A. Karch, Phys. Rev. D 62 (2000) 046008.

[13] M. Gremm, Phys. Lett. B 478 (2000) 434; Phys. Rev. D 62 (2000) 044017; K. Ghoroku and M. Yahiro, Instability of thick brane worlds, arXiv:hep-th/0305150; A. Kehagias and K. Tamvakis, Mod. Phys. Lett. A 17 (2002) 1767; Phys. Lett. B 504 (2001) 38; M. Giovannini, Phys. Rev. D 64 (2001) 064023; Phys. Rev. D 65 (2002) 064008; S. Kobayashi, K. Koyama and J. Soda, Phys. Rev. D 65 (2002) 064014. 
[14] C. Csaki, J. Erlich, T. Hollowood and Y. Shirman, Nucl. Phys. B 581 (2000) 309.

[15] A. Campos, Phys. Rev. Lett. 88 (2002) 141602.

[16] A. Wang, Phys. Rev. D 66 (2002) 024024.

[17] R. Emparan, R. Gregory and C. Santos, Phys. Rev. D 63 (2001) 104022; A. Melfo, N. Pantoja and A. Skirzewski, Phys. Rev. D 67 (2003) 105003; K.A. Bronnikov and B.E. Meierovich, Grav. Cosmol. 9 (2003) 313; O. Castillo-Felisola, A. Melfo, N. Pantoja and A. Ramirez, Phys. Rev. D 70 (2004) 104029; T.R. Slatyer and R.R. Volkas, JHEP 0704 (2007) 062; N. BarbosaCendejas, A. Herrera-Aguilar, U. Nucamendi, C. Schubert, I. Quiros and M.A. Reyes Santos, AIP Conf. Proc. 1026 (2008) 146.

[18] V. Dzhunushaliev, V. Folomeev, D. Singleton and S. Aguilar-Rudametkin, Phys. Rev. D 77 (2008) 044006; V. Dzhunushaliev, V. Folomeev, K. Myrzakulov and R. Myrzakulov, Gen. Rel. Grav. 41 (2009) 131; D. Bazeia, C. Furtado and A.R. Gomes, JCAP 0402 (2004) 002; D. Bazeia, F.A. Brito and L. Losano, JHEP 0611 (2006) 064; D. Bazeia, A.R. Gomes, L. Losano and R. Menezes, Phys. Lett. B 671 (2009) 402.

[19] M. Sarrazin and F. Petit, Equivalence between domain-walls and "non-commutative" twosheeted spacetimes, Model-independent matter swapping between branes, arXiv:0903.2498[hepth]; K. Farakos, N.E. Mavromatos and P. Pasipoularides, Asymmetrically Warped Brane Models, Bulk Photons and Lorentz Invariance, arXiv:0902.1243[hep-th]; D. Bazeia, A.R. Gomes and L. Losano, Int. J. Mod. Phys. A 2420091135.

[20] V. Dzhunushaliev, V. Folomeev and M. Minamitsuji, Thick brane solutions, arXiv:0904.1775[gr-qc].

[21] N. Sasakura, JHEP 0202 (2002) 026; I. Olasagasti and K. Tamvakis, Phys. Rev. D 68 (2003) 064016.

[22] M. Minamitsuji, W. Naylor and M. Sasaki, Nucl. Phys. B 737 (2006) 121.

[23] V. Dzhunushaliev, V. Folomeev and M. Minamitsuji, Phys. Rev. D 79 (2009) 024001, arXiv:0809.4076[gr-qc].

[24] S. Aguilar and D. Singleton, Phys. Rev. D 73 (2006) 085007; M. Gogberashvili, P. Midodashvili and D. Singleton, JHEP 0708 (2007) 033.

[25] B. Bajc and G. Gabadadze, Phys. Lett. B 474 (2000) 282.

[26] Y.-X. Liu, X.-H. Zhang, L.-D. Zhang and Y.-S. Duan, JHEP 0802 (2008) 067, arXiv:0708.0065[hep-th].

[27] Y. Grossman and N. Neubert, Phys. Lett. B 474 (2000) 361;

[28] R. Koley and S. Kar, Phys. Lett. B 623 (2005) 244; [Erratum ibid. 631 (2005) 199; A. Melfo, N. Pantoja and J.D. Tempo, Phys. Rev. D 73 (2006) 044033; R. Davies, D.P. George and R.R. Volkas, Phys. Rev. D 77 (2008) 124038.

[29] S. Ichinose, Phys. Rev. D 66 (2002) 104015.

[30] C. Ringeval, P. Peter and J.P. Uzan, Phys. Rev. D 65 (2002) 044016.

[31] S. Randjbar-Daemi and M. Shaposhnikov, Phys. Lett. B 492 (2000) 361. 
[32] R. Koley and S. Kar, Class. Quantum Grav. 22 (2005) 753.

[33] S.L. Dubovsky, V.A. Rubakov and P.G. Tinyakov, Phys. Rev. D 62 (2000) 105011.

[34] Y. Brihaye and T. Delsate, Phys. Rev. D 78 (2008) 025014.

[35] O. Arias, R. Cardenas and I. Quiros, Nucl. Phys. B 643 (2002) 187; N. Barbosa-Cendejas and A. Herrera-Aguilar, JHEP 0510 (2005) 101; N. Barbosa-Cendejas and A. Herrera-Aguilar, Phys. Rev. D 73 (2006) 084022.

[36] N. Barbosa-Cendejas, A. Herrera-Aguilar, M. A. ReyesSantos and C. Schubert, Phys. Rev. D 77 (2008) 126013; N. Barbosa-Cendejas, A. Herrera-Aguilar, U. Nucamendi and I. Quiros, Mass hierarchy and mass gap on thick branes with Poincare symmetry, arXiv:0712.3098[hepth].

[37] Y.-X. Liu, L.-D. Zhang, S.-W. Wei and Y.-S. Duan, JHEP 0808 (2008) 041, arXiv:0803.0098[hep-th].

[38] C.A.S. Almeida, R. Casana, M.M. Ferreira Jr. and A.R. Gomes, Phys. Rev. D 79 (2009) 125022, arXiv:0901.3543 hep-th].

[39] D. Bazeia, M.J. dos Santos and R.F. Ribeiro, Phys. Lett. A 20884 (1995); D. Bazeia, J.R. Nascimento, R.F. Ribeiro and D. Toledo, J. Phys. A 30, 8157 (1997); D. Bazeia, F.A. Brito, and H. Boschi-Filho, JHEP 04 (1999) 028; D. Bazeia and A.R. Gomes, JHEP 05 (2004) 012.

[40] Y.-X. Liu, Z.-H. Zhao, S.-W. Wei and Y.-S. Duan, JCAP 0902 (2009) 003, arXiv:0901.0782[hep-th].

[41] G. Goetz, J. Math. Phys. 31 (1990) 2683.

[42] R. Gass and M. Mukherjee, Phys. Rev. D 60 (1999) 065011.

[43] R. Guerrero, A. Melfo and N. Pantoja, Phys. Rev. D 65 (2002) 125010.

[44] R. Casadio and A. Gruppuso, Phys. Rev. D64 (2001) 025020.

[45] R. Casadio, A. Gruppuso and G. Venturi, Phys. Lett. B 495 (2000) 378.

[46] Y.-X. Liu, L.-D. Zhang, L.-J. Zhang and Y.-S. Duan, Phys. Rev. D 78 (2008) 065025, arXiv:0804.4553[hep-th].

[47] Y.X. Liu, L. Zhao and Y.S. Duan, JHEP 0704 (2007) 097, arXiv:hep-th/0701010.

[48] S.L. Parameswaran, S. Randjbar-Daemi and A. Salvio, Nucl. Phys. B 767 (2007) 54, arXiv:hep-th/0608074.

[49] Y.X. Liu, L. Zhao, X.H. Zhang and Y.S. Duan, Nucl. Phys. B 785 (2007) 234, arXiv:0704.2812[hep-th].

[50] G. Gibbons, K. Maeda and Y. Takamizu, Phys. Lett. B 647 (2007) 1.

[51] Y.Q. Wang, T.Y. Si, Y.X. Liu and Y.S. Duan, Mod. Phys. Lett. A 20 (2005) 3045; Y.S. Duan, Y.X. Liu and Y.Q. Wang, Mod. Phys. Lett. A 21 (2006) 2019; Y.X. Liu, Y.Q. Wang and Y.S. Duan, Commun. Theor. Phys. 48 (2007) 675.

[52] R. Koley, J. Mitra and S. SenGupta, Phys. Rev. D 78 (2008) 045005; R. Koley, Localization of fields on brane, Published in Proceedings of the Workshop on "Physics of warped extra dimensions' (February 21-23, 2008), Page 19-25, arXiv:0812.1423[hep-th]; X.-H. Zhang, Y.-X. 
Liu and Y.-S. Duan, Mod. Phys. Lett. A 23 (2008) 2093, arXiv:0709.1888[hep-th]; L. Zhao, Y.-X. Liu and Y.i-S. Duan, Mod. Phys. Lett. A 23 (2008) 1129, arXiv:0709.1520[hep-th].

[53] D. Bazeia, F.A. Brito and R.C. Fonseca, Fermion states on domain wall junctions and the flavor number, submitted to Eur. Phys. J. C, arXiv:0809.3048[hep-th]; P. Koroteev and M. Libanov, Phys. Rev. D 79 (2009) 045023, arXiv:0901.4347[hep-th]; A. Flachi and M. Minamitsuji, Phys. Rev. D 79 (2009) 104021, arXiv:0903.0133[hep-th].

[54] Y. Kodama, K. Kokubu and N. Sawado, Phys. Rev. D 79 (2009) 065024, arXiv:0812.2638[hepth].

[55] R. Gregory, V.A. Rubakov and S.M. Sibiryakov, Phys. Rev. Lett. 84 (2000) 5928, arXiv:hep-th/0002072.

[56] J.F. Vazquez-Poritz, JHEP 12 (2001) 030, arXiv:hep-th/0110299.

[57] S. Mouslopoulos, JHEP 05 (2001) 038, arXiv: hep-th/0103184.

[58] P.M. Llatas, Phys. Lett. B 514, 139 (2001). 